\title{
Frações químicas e oxidáveis da matéria orgânica do solo sob diferentes sistemas de manejo, em Latossolo Vermelho
}

\author{
Jean Sérgio Rosset ${ }^{(1)}$, Maria do Carmo Lana ${ }^{(2)}$, Marcos Gervasio Pereira(3), Jolimar Antonio Schiavo ${ }^{(4)}$, \\ Leandro Rampim ${ }^{(5)}$ e Marcos Vinícius Mansano Sarto(6)
}

\begin{abstract}
(1)Universidade Estadual de Mato Grosso do Sul (UEMS), BR 163, Km 20,2, CEP 79980-000 Mundo Novo, MS, Brasil. E-mail: rosset@uems.br (2)Universidade Estadual do Oeste do Paraná, Centro de Ciências Agrárias, Rua Pernambuco, no 1.777, Caixa Postal 1008, Centro, CEP 85960-000 Marechal Cândido Rondon, PR, Brasil. E-mail: maria.lana@unioeste.br (3)Universidade Federal Rural do Rio de Janeiro, Departamento de Solos, BR 465, Km 7, CEP 23890-000 Seropédica, RJ, Brasil. E-mail: gervasio@ufrrj.br (4) UEMS, Rodovia Aquidauana, Km 12, Zona Rural, Caixa Postal 25, CEP 79200-000 Aquidauana, MS, Brasil. E-mail: schiavo@uems.br (5)Universidade Estadual do Centro-Oeste, Campus Cedeteg, Guarapuava, PR, Brasil. E-mail: rampimleandro@yahoo.com.br ${ }^{(6)}$ Universidade Estadual Paulista, Faculdade de Ciências Agronômicas, Caixa Postal 237, CEP 18610-307 Botucatu, SP, Brasil. E-mail: marcos_sarto@hotmail.com
\end{abstract}

Resumo - O objetivo deste trabalho foi avaliar a qualidade do solo a partir das frações químicas e oxidáveis da matéria orgânica (MOS) em áreas com diferentes sistemas de manejo. O trabalho foi realizado no Município de Guaíra, no Oeste do Paraná, em áreas com diferentes tempos de adoção do sistema plantio direto (SPD, 6, 14 e 22 anos), com sucessão das culturas soja (verão) e milho/trigo (inverno), além de áreas com 12 anos de SPD - no mesmo arranjo de sucessão - mais quatro anos de cultivo de braquiária (Urochloa ruziziensis) consorciada com milho (M+B) e com pastagem e mata nativa. As frações químicas de ácido fúlvico (AF), ácido húmico (AH) e humina (HUM) foram avaliadas, bem como as frações oxidáveis da MOS (F1, F2, F3 e F4), o carbono total (CT) e o estoque de carbono (C) nas frações húmicas. Os teores de CT aumentaram com o tempo de adoção do SPD, principalmente entre $\mathrm{SPD}_{6}$ e $\mathrm{SPD}_{22}$. Verificou-se predomínio da fração HUM e movimentação de $\mathrm{AH}$ e $\mathrm{AF}$ na camada de $0,0-0,4 \mathrm{~m}$ de profundidade. As frações oxidáveis apresentaram equilíbrio na distribuição do conteúdo de C, com maiores teores nas frações mais lábeis, em superfície. O cultivo com sucessão de culturas contribui para o aumento das frações mais lábeis, mas não para o aumento das frações mais recalcitrantes do C.

Termos para indexação: Urochloa ruziziensis, carbono lábil, estoque de carbono, fracionamento da matéria orgânica, qualidade do solo, substâncias húmicas.

\section{Chemical and oxidizable fractions of soil organic matter under different management systems in an Oxisol}

\begin{abstract}
The objective of this work was to evaluate soil quality from soil organic matter (SOM) chemical and oxidizable fractions in areas with different management systems. The study was carried out in the municipality of Guaira, in the west of the state of the Paraná, Brazil, in areas with different adoption times of the no-tillage system (NT; 6, 14, and 22 years), with succession scheme of soybean (summer) and corn/wheat (winter) crops, besides areas with 12 years under NT - in the same succession scheme - plus four years of Urochloa ruziziensis intercropped with maize $(\mathrm{M}+\mathrm{U})$, and with pasture and native forest. The chemical fractions of fulvic acid (FA), humic acid (HA), and humin (HUM) were evaluated, as well as the oxidizable fractions of MOS (F1, F2, F3, and F4), total carbon (TC), and carbon storage (C) in the humic fractions. Total carbon contents increased with the increasing adoption of time of SPD, especially from $\mathrm{NT}_{6}$ to $\mathrm{NT}_{22}$. Humin fraction predominance was observed, with movement of HA and FA in the $0.0-0.4 \mathrm{~m}$ soil depth. The oxidizable $\mathrm{C}$ fractions showed equilibrium in the distribution of $\mathrm{C}$ contents, with higher levels in the more labile fractions, at surface. The cultivation with succession of crops contributes to the increase in labile fractions, but not to the increase of the more recalcitrant fractions of C.

Index terms: Urochloa ruziziensis, labile carbon, carbon storage, organic matter fractioning, soil quality, humic substances.
\end{abstract}

\section{Introdução}

Sistemas de manejo conservacionistas, como o sistema plantio direto (SPD), capazes de manter ou até mesmo incrementar frações de carbono orgânico total (COT) no solo, contribuem para manutenção da capacidade produtiva e para mitigação da emissão de $\mathrm{CO}_{2}$ para a atmosfera (Foley et al., 2005). 
A compreensão da dinâmica da matéria orgânica do solo (MOS) em sistemas de produção permite subsidiar o estabelecimento de estratégias de manejo que garantam incremento ou manutenção da qualidade do solo ao longo do tempo (Rossi et al., 2011). A MOS pode ser alterada em maior ou menor grau, e é um dos atributos mais sensíveis ao manejo dos solos (Barreto et al., 2008). Entretanto, em algumas situações, não é possível identificar impacto do manejo do solo somente com análise da variação dos teores de COT (Barreto et al., 2011). Neste caso, é importante que se conheça a forma de distribuição dos diferentes compartimentos que compõem a MOS, como o carbono (C) das frações húmicas (Bezerra et al., 2013) e das frações oxidáveis (Rangel et al., 2008), para que esses atributos possam ser utilizados como indicadores de qualidade do solo ao longo do tempo de cultivo (Barreto et al., 2011).

Grande parte da MOS consiste de compostos ácidos não humificados e macromoléculas humificadas. As substâncias húmicas $(\mathrm{SH})$ apresentam representatividade em torno de $85 \%$ a $90 \%$ do COT e são divididas em: ácidos húmicos $(\mathrm{AH})$, ácidos fúlvicos (AF) e humina (HUM) (Guerra et al., 2008). Além das frações húmicas, as frações oxidáveis da MOS (Chan et al., 2001) auxiliam na interpretação da dinâmica do $\mathrm{C}$ no solo. Elas são baseadas nos graus de oxidação do $\mathrm{C}$ e apresentam quatro frações (F1, F2, F3 e F4), as duas primeiras relacionadas à disponibilidade de nutrientes e à formação e estabilização de macroagregados (Barreto et al., 2011); e as duas últimas relacionadas a compostos de maior estabilidade química e peso molecular, comuns nas frações humificadas da MOS (Rangel et al., 2008) de maior tempo de residência no solo (Chan et al., 2001).

Rossi et al. (2011) e Loss et al. (2013) verificaram que o $\mathrm{C}$ das frações húmicas é útil para identificar mudanças promovidas pelos sistemas de manejo em solos do Cerrado goiano. Também na região dos cerrados, Guareschi et al. (2013a, 2013b) verificaram elevada utilidade das frações oxidáveis para avaliar a qualidade dos solos em estudo com cronossequência em SPD. Contudo, trabalhos de longo prazo ainda são importantes para uma compreensão mais abrangente e consistente do tema.

O objetivo deste trabalho foi avaliar a qualidade do solo a partir das frações químicas e oxidáveis da matéria orgânica (MOS) em áreas com diferentes sistemas de manejo.

\section{Material e Métodos}

As amostras de solo foram coletadas em sistemas de manejo explorados comercialmente, com diferentes tempos de implantação, e conduzidos em propriedades rurais no Município de Guaíra, no Oeste do Paraná, Brasil. O clima da região é subtropical (Cfa), segundo classificação de Köppen (Caviglione et al., 2000), e o solo das áreas de estudo é classificado como Latossolo Vermelho Eutroférrico típico, textura muito argilosa (Santos et al., 2013).

Foram avaliadas cinco áreas manejadas, além de uma área de referência (mata nativa - Floresta Estacional Semi Decidual da Mata Atlântica) que não sofreu ação antrópica, em delineamento inteiramente casualizado. Foram avaliadas áreas com: sistema de plantio direto por 6 (fase de transição, $\mathrm{SPD}_{6}$ ), 14 (fase de consolidação, $\mathrm{SPD}_{14}$ ) e 22 anos (fase de manutenção, $\mathrm{SPD}_{22}$ ). $\mathrm{O}$ cultivo se deu com sucessão de culturas soja (verão) e milho/trigo (inverno). Além dessas, avaliouse também uma área com 12 anos de SPD, no mesmo sistema de sucessão, e mais quatro anos com introdução de braquiária (Urochloa ruziziensis) em consórcio com o milho nos cultivos de inverno (fase de consolidação, M+B) (Anghinoni, 2007), e outra área de pastagem permanente coast-cross (Cynodon dactylon) (38 anos), com lotação animal de 3,5 UA ha ${ }^{-1}$, sem sinais visíveis de degradação (Figura 1). A caracterização química e a granulométrica das áreas avaliadas encontram-se descritas na Tabela 1.

Em todas as áreas sob SPD, as adubações utilizadas nos últimos 5 anos de cultivo em sucessão das culturas de soja, milho/trigo foram, respectivamente, $270 \mathrm{~kg}$ ha $^{-1}$ de 02-20-18 e inoculação com Bradyrhizobium japonicum e $270 \mathrm{~kg} \mathrm{ha}^{-1}$ de 10-15-15, além da aplicação de calcário a cada 4 anos, na dose de $1,7 \mathrm{Mg} \mathrm{ha}{ }^{-1}$, usualmente utilizada na região, com exceção da área de $\mathrm{SPD}_{14}$, que, após a conversão do sistema de preparo convencional (SPC) para SPD, em 1998, não recebeu correção do solo. Na área cultivada com pastagem, não foram realizadas correção ou adubação durante todo o período em que a espécie ficou instalada na área.

Em cada área de estudo, foram demarcadas cinco glebas de $400 \mathrm{~m}^{2}$, cada uma representando uma repetição, nas quais foram realizadas as coletas das amostras de terra. Cada amostra composta foi representada por dez amostras simples, dentro dos seis 
sistemas de manejo, nas camadas de $0-0,05,0,05-0,10$, $-0,10-0,20$ e $0,20-0,40 \mathrm{~m}$. Após a coleta, as amostras foram secas ao ar, destorroadas e passadas por peneira $2 \mathrm{~mm}$, para obtenção da terra fina seca ao ar (TFSA). Também foram coletadas amostras indeformadas com auxílio de anel volumétrico de $46,2 \mathrm{~cm}^{3}$, com cinco repetições em todas as áreas e camadas.

O carbono total (CT) foi determinado por combustão seca em analisador CHNS (Elementar Analysensysteme GmbH, Hanau, Alemanha). O fracionamento químico da MOS foi realizado segundo Swift (1996) com adaptações de Benites et al. (2003). A partir das análises de $\mathrm{C}$ dos ácidos húmicos $(\mathrm{AH})$,

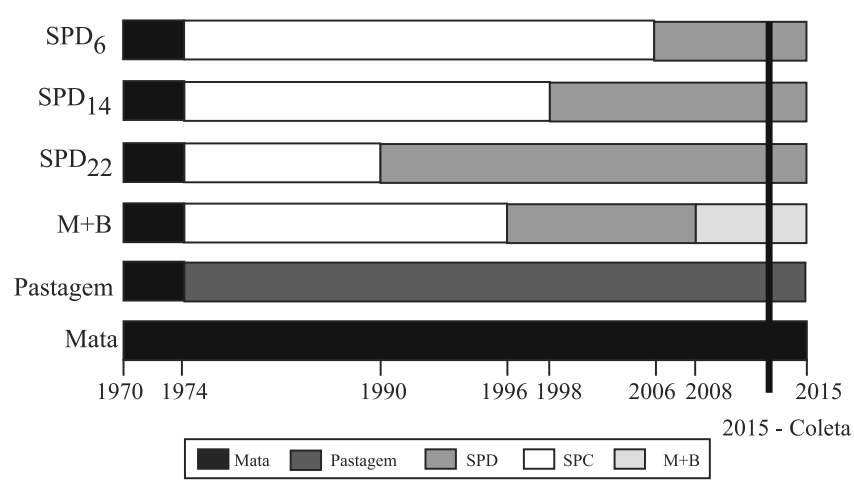

Figura 1. Histórico de uso das áreas avaliadas, com as respectivas datas de implantação, altitude e localização geográfica de cada sistema de manejo. SPD, sistema plantio direto; SPC, sistema de preparo convencional; e $\mathrm{M}+\mathrm{B}$, sistema plantio direto em consórcio com a braquiária (Urochloa ruziziensis).

Tabela 1. Caracterização química e granulométrica do solo na camada de 0-0,20 m nos diferentes sistemas de manejo.

\begin{tabular}{|c|c|c|c|c|c|c|c|c|c|c|}
\hline \multirow[t]{2}{*}{$\begin{array}{l}\text { Sistema } \\
\text { de manejo }\end{array}$} & \multirow[t]{2}{*}{$\begin{array}{c}\mathrm{pH} \\
\left(\mathrm{CaCl}_{2}\right)\end{array}$} & \multirow{2}{*}{$\begin{array}{c}\mathrm{P} \\
\left(\mathrm{mg} \mathrm{dm}^{-3}\right)\end{array}$} & $\mathrm{H}+\mathrm{Al}$ & $\mathrm{Al}$ & $\mathrm{Ca}$ & $\mathrm{Mg}$ & \multirow{2}{*}{ K } & \multirow[t]{2}{*}{ Areia } & \multirow[t]{2}{*}{ Silte } & \multirow{2}{*}{$\begin{array}{l}\text { Argila } \\
\text {------ }\end{array}$} \\
\hline & & & ----- & (c) & 1 & $-3)$ & & & & \\
\hline $\mathrm{SPD}_{6}$ & 5,5 & 14,8 & 3,3 & 0,0 & 4,3 & 1,4 & 0,3 & 160 & 213 & 627 \\
\hline $\mathrm{SPD}_{14}$ & 4,9 & 22,2 & 4,7 & 0,4 & 2,5 & 0,6 & 0,2 & 139 & 164 & 697 \\
\hline $\mathrm{SPD}_{22}$ & 5,7 & 20,1 & 3,8 & 0,0 & 5,4 & 1,1 & 0,4 & 98 & 271 & 631 \\
\hline $\mathrm{M}+\mathrm{B}$ & 5,6 & 19,0 & 2,9 & 0,0 & 4,9 & 0,8 & 0,3 & 167 & 212 & 621 \\
\hline Pastagem & 6,3 & 9,6 & 2,6 & 0,0 & 2,2 & 0,6 & 0,2 & 86 & 295 & 619 \\
\hline Mata & 6,3 & 6,9 & 2,4 & 0,0 & 7,3 & 0,9 & 0,3 & 116 & 268 & 616 \\
\hline
\end{tabular}

$\mathrm{SPD}_{6}$, sistema plantio direto por seis anos; $\mathrm{SPD}_{14}$, por 14 anos; $\mathrm{SPD}_{22}$, por 22 anos; todos com sucessão soja, milho/trigo. M+B, SPD por 12 anos (com mesmo esquema de sucessão), mais quatro anos de consórcio entre Urochloa ruziziensis e milho nos cultivos de inverno. Pastagem de coast-cross (Cynodon dactylon) por 38 anos; e Mata representa a mata nativa (Rosset et al., 2014). ácidos fúlvicos (AF), extrato alcalino (EA) $(\mathrm{EA}=$ $\mathrm{AH}+\mathrm{AF}$ ) e humina (HUM), foram calculadas as relações $\mathrm{AH} / \mathrm{AF}$ e EA/HUM, para verificação dos processos de humificação da MOS. Posteriormente, foram calculados os estoques de $\mathrm{C}$ das $\mathrm{SH}$, conforme o método da massa equivalente (Ellert \& Bettany, 1995; Sisti et al., 2004). Para verificar tendências de acúmulo ou perda de $\mathrm{C}$ das frações húmicas na seção de $0-0,40$ $\mathrm{m}$, foi calculada a variação do estoque de C (EC) de cada fração, em comparação à da mata (referência) $\left(\triangle \mathrm{EC}, \mathrm{Mg} \mathrm{ha}^{-1} \mathrm{~cm}^{-1}\right)$.

O fracionamento da porção oxidável da MOS de acordo com os diferentes graus de oxidação foi efetuado segundo Chan et al. (2001), com separação das frações de C em F1, F2, F3 e F4, em que o C na fração $\mathrm{F} 1$ é considerado como carbono lábil $\left(\mathrm{C}_{\mathrm{L}}\right)$, e o carbono não lábil $\left(\mathrm{C}_{\mathrm{NL}}\right)$ é obtido por diferença: $\mathrm{C}_{\mathrm{NL}}=$ COT $-\mathrm{C}_{\mathrm{L}}$. Posteriormente, calculou-se a relação $\mathrm{C}_{\mathrm{L}} /$ $\mathrm{COT}$, bem como as relações entre as frações $\mathrm{F} 1 / \mathrm{F} 4$ e $\mathrm{F} 1+\mathrm{F} 2 / \mathrm{F} 3+\mathrm{F} 4$.

Os resultados foram analisados quanto à normalidade e homogeneidade de variâncias e posteriormente submetidos à análise de variância. As médias foram comparadas pelo teste de Tukey, a $5 \%$ de probabilidade, com uso do programa Genes (Cruz, 2006). Como análise complementar, utilizouse a análise canônica (multivariada), a qual envolveu as variáveis das frações químicas e oxidáveis da MOS separadamente, para redução do conjunto de dados em combinações lineares, tendo-se gerado os escores das duas primeiras variáveis canônicas que explicavam mais de $80 \%$ da variação total (Cruz \& Regazzi, 1994), com os escores projetados em gráficos bidimensionais. Além dessa técnica, utilizou-se ainda o método de agrupamento de Tocher modificado, com o propósito de discriminar os tratamentos de maior similaridade, e também para agrupar os diferentes tipos de manejo a partir da matriz de distância generalizada de Mahalanobis. Os gráficos com base na análise canônica foram gerados, e os grupos, formados por meio do agrupamento de Tocher modificado, com uso do programa Genes (Cruz, 2006).

\section{Resultados e Discussão}

Os teores de CT aumentaram com o tempo de adoção do SPD, principalmente entre as áreas de $\mathrm{SPD}_{6}$ e $\mathrm{SPD}_{22}$, nas camadas de $0-0,05$ e $0,05-0,10 \mathrm{~m}$,

Pesq. agropec. bras., Brasília, v.51, n.9, p.1529-1538, set. 2016 DOI: 10.1590/S0100-204X2016000900052 
e $\mathrm{SPD}_{14}$ e $\mathrm{SPD}_{22}$, na camada de $0-0,05 \mathrm{~m}$ (Tabela 2). Em geral, maiores modificações nos teores de CT são observadas após 10 a 15 anos da conversão do sistema de preparo convencional (SPC) para o SPD (Carvalho et al., 2009). Sob o mesmo sistema de sucessão de culturas, em Latossolo Vermelho Distrófico no Sul do Brasil, Sisti et al. (2004) não verificaram acúmulos significativos de COT na camada de $0-0,3 \mathrm{~m}$ após 13 anos sob SPD. Esse resultado também foi relatado por Guareschi et al. (2013b) em cronossequência de 3, 15 e 20 anos de SPD sob Latossolo Vermelho Distroférrico no Cerrado goiano.

Na camada de $0-0,05 \mathrm{~m}$, a área de mata apresentou maior teor de CT $\left(40,78 \mathrm{~g} \mathrm{~kg}^{-1}\right)$, seguida das áreas de pastagem $\left(31,56 \mathrm{~g} \mathrm{~kg}^{-1}\right)$ e $\operatorname{SPD}_{22}\left(24,02 \mathrm{~g} \mathrm{~kg}^{-1}\right)$, que foram significativamente diferentes entre si e das demais áreas avaliadas. Nas duas últimas camadas, a área de pastagem apresentou os maiores teores de CT. Essa tendência também foi relatada por Loss et al. (2014), em área de pastagem e SPD com 15 anos de sucessão soja/azevém. Esse padrão de resposta destaca a contribuição do sistema radicular das gramíneas perenes em acumular $\mathrm{C}$ em profundidade, uma vez que a área do estudo está ocupada com pastagem permanente há 38 anos, o que favorece a proteção de C no interior dos agregados (Loss et al., 2011).

De modo geral, os teores de CT diminuíram em profundidade, de maneira similar à relatada por Bezerra et al. (2013), em SPD, com integração lavourapecuária e mata nativa sob Latossolo Vermelho Distrófico no Estado de Goiás. Essa diminuição dos teores de $\mathrm{C}$ evidencia a importância das entradas de $\mathrm{C}$ na camada mais superficial, em comparação às camadas de $0,05-0,10,0,10-0,20$ e $0,20-0,40 \mathrm{~m}$, fato comum em sistemas sem revolvimento intensivo do solo (Bezerra et al., 2013), como o de SPD consolidado (Guareschi et al., 2013a).

Foram observadas perdas percentuais de CT de 54, 49, 41, 54 e 23\%, respectivamente nas áreas de $\mathrm{SPD}_{6}$, $\mathrm{SPD}_{14}, \mathrm{SPD}_{22}, \mathrm{M}+\mathrm{B}$ e pastagem, em comparação com a mata, na camada de $0-0,05 \mathrm{~m}$. Com exceção da área de pastagem, as modificações nessa camada foram dependentes do tempo que as áreas ficaram sujeitas ao revolvimento intensivo do solo sob SPC, após o desmatamento em 1974.

Similarmente ao observado para o CT, verificaram-se maiores teores de $\mathrm{C}$ das frações húmicas na camada de
0-0,05 m, principalmente para C-HUM, $\left(28,13 \mathrm{~g} \mathrm{~kg}^{-1}\right)$, na área de mata (Tabela 2). Os teores de C-HUM foram superiores aos das frações $\mathrm{AF}$ e $\mathrm{AH}$, o que está em conformidade ao observado na literatura em solos com maiores teores de argila (Bezerra et al., 2013; Caetano et al., 2013; Campos et al., 2013; Guareschi et al., 2013a), e indica maior interação dessa fração recalcitrante da MOS com a fração argila em solos tropicais (Assis et al., 2006). Corroboram essa asserção os valores da relação EA/HUM inferiores à unidade (Tabela 2), padrão também observado para os estoques de C-HUM (Tabela 3).

Em todas as áreas, a fração HUM representou, em média, $64,62,59$ e $58 \%$ do COT, nas camadas de $0-0,05$,

Tabela 2. Teores $\left(\mathrm{g} \mathrm{kg}^{-1}\right)$ de carbono total (CT) e das frações humificadas da matéria orgânica do solo nos diferentes sistemas de manejo na região Oeste do Paraná ${ }^{(1)}$.

\begin{tabular}{|c|c|c|c|c|c|c|c|}
\hline $\begin{array}{l}\text { Sistema de } \\
\text { manejo }\end{array}$ & $\mathrm{CT}$ & $\mathrm{C}-\mathrm{AF}$ & $\mathrm{C}-\mathrm{AH}$ & C-HUM & EA & $\mathrm{AH} / \mathrm{AF}$ & EA/HUM \\
\hline & \multicolumn{7}{|c|}{$0-0,05 \mathrm{~m}$} \\
\hline $\mathrm{SPD}_{6}$ & $18,78 \mathrm{~d}$ & $2,83 \mathrm{c}$ & $2,82 \mathrm{c}$ & $11,14 \mathrm{e}$ & $5,65 \mathrm{~b}$ & $1,01 b c$ & $0,51 \mathrm{a}$ \\
\hline $\mathrm{SPD}_{14}$ & $20,94 d$ & $2,36 \mathrm{~d}$ & $2,12 \mathrm{~d}$ & $12,37 d$ & $4,48 \mathrm{c}$ & $0,90 \mathrm{~cd}$ & $0,36 \mathrm{c}$ \\
\hline $\mathrm{SPD}_{22}$ & $24,02 \mathrm{c}$ & $4,24 \mathrm{a}$ & $3,72 b$ & $15,15 \mathrm{c}$ & $7,96 a$ & $0,88 \mathrm{~cd}$ & $0,53 \mathrm{a}$ \\
\hline $\mathrm{M}+\mathrm{B}$ & $18,96 \mathrm{~d}$ & $3,21 b c$ & $2,59 \mathrm{c}$ & $12,52 \mathrm{~d}$ & $5,80 \mathrm{~b}$ & $0,81 \mathrm{~d}$ & $0,46 b$ \\
\hline Pastagem & $31,56 \mathrm{~b}$ & $3,26 b$ & $4,43 a$ & $21,98 b$ & $7,69 a$ & $1,36 \mathrm{a}$ & $0,35 \mathrm{c}$ \\
\hline Mata & $40,78 \mathrm{a}$ & $3,87 \mathrm{a}$ & $4,15 \mathrm{a}$ & $28,13 \mathrm{a}$ & $8,02 \mathrm{a}$ & $1,07 \mathrm{~b}$ & $0,29 d$ \\
\hline \multirow[t]{2}{*}{$\mathrm{CV}(\%)$} & 4,9 & 6,2 & 6,2 & 3,0 & 3,1 & 8,8 & 5,2 \\
\hline & \multicolumn{7}{|c|}{$0,05-0,10 \mathrm{~m}$} \\
\hline $\mathrm{SPD}_{6}$ & $15,90 \mathrm{~d}$ & $2,60 \mathrm{~cd}$ & $2,55 \mathrm{~b}$ & $8,82 \mathrm{~d}$ & $5,15 \mathrm{bc}$ & $0,98 \mathrm{a}$ & $0,58 \mathrm{~b}$ \\
\hline $\mathrm{SPD}_{14}$ & $16,76 \mathrm{~cd}$ & $1,95 \mathrm{e}$ & $1,68 \mathrm{c}$ & $9,83 \mathrm{c}$ & $3,63 \mathrm{~d}$ & $0,86 \mathrm{~b}$ & $0,37 \mathrm{c}$ \\
\hline $\mathrm{SPD}_{22}$ & $18,24 b c$ & $4,02 \mathrm{a}$ & $3,01 \mathrm{a}$ & $10,55 \mathrm{c}$ & $7,03 \mathrm{a}$ & $0,75 b$ & $0,67 \mathrm{a}$ \\
\hline $\mathrm{M}+\mathrm{B}$ & $17,32 \mathrm{~cd}$ & $2,97 b$ & $2,34 b$ & $10,00 \mathrm{c}$ & $5,31 \mathrm{~b}$ & $0,79 b$ & $0,53 b$ \\
\hline Pastagem & $19,60 \mathrm{~b}$ & $2,30 \mathrm{~d}$ & $2,40 \mathrm{~b}$ & $13,91 b$ & $4,70 \mathrm{c}$ & $1,05 \mathrm{a}$ & $0,34 \mathrm{c}$ \\
\hline Mata & $26,04 a$ & $2,84 b c$ & $2,21 b$ & $18,78 \mathrm{a}$ & $5,05 \mathrm{bc}$ & $0,78 \mathrm{~b}$ & $0,27 \mathrm{~d}$ \\
\hline \multirow[t]{2}{*}{ CV (\%) } & 4,9 & 6,3 & 8,1 & 3,1 & 5,2 & 7,0 & 7,1 \\
\hline & \multicolumn{7}{|c|}{$0,10-0,20 \mathrm{~m}$} \\
\hline $\mathrm{SPD}_{6}$ & $12,44 \mathrm{c}$ & $2,48 b$ & $1,71 \mathrm{~b}$ & $6,73 c$ & $4,19 \mathrm{c}$ & $0,70 \mathrm{~b}$ & $0,62 b$ \\
\hline $\mathrm{SPD}_{14}$ & $12,72 \mathrm{c}$ & $1,71 \mathrm{c}$ & $1,17 \mathrm{c}$ & $7,24 \mathrm{c}$ & $2,88 \mathrm{e}$ & $0,69 b$ & $0,40 \mathrm{~d}$ \\
\hline $\mathrm{SPD}_{22}$ & $14,76 \mathrm{bc}$ & $3,60 \mathrm{a}$ & $2,34 \mathrm{a}$ & $8,58 \mathrm{~b}$ & $5,94 \mathrm{a}$ & $0,65 \mathrm{bc}$ & $0,69 \mathrm{a}$ \\
\hline $\mathrm{M}+\mathrm{B}$ & $15,82 b$ & $2,84 b$ & $1,67 b$ & $8,63 b$ & $4,51 b c$ & $0,59 \mathrm{c}$ & $0,52 \mathrm{c}$ \\
\hline Pastagem & $19,82 \mathrm{a}$ & $2,57 b$ & $2,15 \mathrm{a}$ & $13,11 \mathrm{a}$ & $4,72 b$ & $0,84 a$ & $0,36 \mathrm{~d}$ \\
\hline Mata & $14,94 \mathrm{bc}$ & $2,48 \mathrm{~b}$ & $0,96 \mathrm{c}$ & $9,48 \mathrm{~b}$ & $3,44 d$ & $0,39 \mathrm{~d}$ & $0,36 \mathrm{~d}$ \\
\hline \multirow[t]{2}{*}{ CV (\%) } & 9,7 & 7,8 & 7,9 & 5,3 & 5,9 & 7,3 & 5,8 \\
\hline & \multicolumn{7}{|c|}{$0,20-0,40 \mathrm{~m}$} \\
\hline $\mathrm{SPD}_{6}$ & $9,82 b$ & $2,25 b$ & $1,02 b$ & $5,23 \mathrm{c}$ & $3,27 \mathrm{c}$ & $0,46 b c$ & $0,62 b$ \\
\hline $\mathrm{SPD}_{14}$ & $10,42 b$ & $1,51 \mathrm{c}$ & $0,61 \mathrm{c}$ & $6,04 \mathrm{bc}$ & $2,12 \mathrm{e}$ & $0,40 \mathrm{c}$ & $0,35 \mathrm{~d}$ \\
\hline $\mathrm{SPD}_{22}$ & $9,90 \mathrm{~b}$ & $3,06 \mathrm{a}$ & $1,36 a$ & $5,25 \mathrm{c}$ & $4,42 \mathrm{a}$ & $0,44 b c$ & $0,84 \mathrm{a}$ \\
\hline $\mathrm{M}+\mathrm{B}$ & $10,64 b$ & $2,55 b$ & $1,31 \mathrm{a}$ & $6,24 b$ & $3,86 \mathrm{~b}$ & $0,52 \mathrm{ab}$ & $0,65 b$ \\
\hline Pastagem & $11,84 \mathrm{a}$ & $2,15 b$ & $1,19 \mathrm{ab}$ & $8,01 \mathrm{a}$ & $3,34 \mathrm{c}$ & $0,55 \mathrm{a}$ & $0,42 \mathrm{~cd}$ \\
\hline Mata & $10,78 \mathrm{~b}$ & $2,14 b$ & $0,67 \mathrm{c}$ & $6,31 \mathrm{~b}$ & $2,80 \mathrm{~d}$ & $0,32 \mathrm{~d}$ & $0,44 \mathrm{c}$ \\
\hline CV (\%) & 4,9 & 10,1 & 9,3 & 6,7 & 6,1 & 9,5 & 7,2 \\
\hline
\end{tabular}

${ }^{(1)}$ Médias seguidas de letras iguais na coluna, em cada camada, não diferem pelo teste de Tukey a 5\% de probabilidade. $\mathrm{C}-\mathrm{AF}$, ácido fúlvico; $\mathrm{C}-\mathrm{AH}$, ácido húmico; e C-HUM, humina; EA, extrato alcalino. 
$0,05-0,10,0,10-0,20$ e $0,20-0,40 \mathrm{~m}$, respectivamente, tendo chegado a $72 \%$ na área de mata, na camada de $0,05-0,10 \mathrm{~m}$. Esses valores estão dentro da faixa comumente observada na literatura e sugerem grau avançado de humificação da MOS - especialmente nas áreas de mata e pastagem -, uma vez que a fração HUM é a fração de grande estabilidade (Fontana et al., 2006), resistente à biodegradação devido à complexação com íons metálicos ou pela formação de compostos argilohúmicos estáveis (Benites et al., 2003; Barreto et al., 2008). As frações AH e AF submetidas a processos de polimerização e mineralização são menos estáveis e apresentam maior movimentação no perfil do solo (Fontana et al., 2006).

A fração $\mathrm{AF}$ apresentou movimentação mais evidente no solo, uma vez que sua representatividade aumentou em profundidade, com valores médios de $13 \%, 15 \%$, $17 \%$ e $22 \%$. Esse padrão também foi evidenciado pelo aumento nos estoques de C-AF (Tabela 3), em todas as áreas, e na relação EA/HUM em profundidade (Tabela 2).

A relação $\mathrm{AH} / \mathrm{AF}$ apresentou valores superiores a unidade, somente nas áreas $\mathrm{SPD}_{6}$, pastagem e mata, na camada de $0-0,05 \mathrm{~m}$, e, no caso da pastagem, também na camada de 0,05-0,10 m (Tabela 2). Os valores de

Tabela 3. Estoque de $\mathrm{C}\left(\mathrm{Mg} \mathrm{ha}^{-1}\right)$ das frações humina (C-HUM), ácido húmico (C-AH) e ácido fúlvico (AF), nos diferentes sistemas de manejo na região Oeste do Paraná(1).

\begin{tabular}{llllllll}
\hline \multirow{2}{*}{$\begin{array}{l}\text { Profundidade } \\
(\mathrm{m})\end{array}$} & \multicolumn{7}{c}{ Sistemas de uso do solo } \\
\cline { 2 - 8 } & $\mathrm{SPD}_{6}$ & $\mathrm{SPD}_{14}$ & $\mathrm{SPD}_{22}$ & $\mathrm{M}+\mathrm{B}$ & Pastagem & Mata & $\mathrm{CV}(\%)$ \\
\hline $0-0,05$ & $4,61 \mathrm{c}$ & $5,12 \mathrm{c}$ & $6,26 \mathrm{c}$ & $5,19 \mathrm{c}$ & $9,11 \mathrm{~b}$ & $11,64 \mathrm{a}$ & 13,0 \\
$0,05-0,10$ & $4,61 \mathrm{~b}$ & $5,39 \mathrm{~b}$ & $6,09 \mathrm{~b}$ & $5,42 \mathrm{~b}$ & $8,09 \mathrm{a}$ & $9,07 \mathrm{a}$ & 11,9 \\
$0,10-0,20$ & $7,94 \mathrm{c}$ & $8,70 \mathrm{bc}$ & $10,00 \mathrm{~b}$ & $9,95 \mathrm{~b}$ & $14,67 \mathrm{a}$ & $10,58 \mathrm{~b}$ & 9,5 \\
$0,20-0,40$ & $11,29 \mathrm{~b}$ & $12,71 \mathrm{~b}$ & $12,06 \mathrm{~b}$ & $13,61 \mathrm{~b}$ & $17,91 \mathrm{a}$ & $13,02 \mathrm{~b}$ & 9,3 \\
$0-0,40$ & $28,45 \mathrm{~d}$ & $31,92 \mathrm{~cd}$ & $34,41 \mathrm{c}$ & $34,17 \mathrm{c}$ & $49,78 \mathrm{a}$ & $44,31 \mathrm{~b}$ & 7,1 \\
\hline & & \multicolumn{7}{c}{ Estoque de C-AH } \\
$0-0,05$ & $1,17 \mathrm{bc}$ & $0,88 \mathrm{c}$ & $1,55 \mathrm{ab}$ & $1,07 \mathrm{c}$ & $1,84 \mathrm{a}$ & $1,72 \mathrm{a}$ & 15,9 \\
$0,05-0,10$ & $1,29 \mathrm{abc}$ & $0,91 \mathrm{c}$ & $1,61 \mathrm{a}$ & $1,20 \mathrm{bc}$ & $1,51 \mathrm{ab}$ & $1,06 \mathrm{c}$ & 16,1 \\
$0,10-0,20$ & $2,06 \mathrm{bc}$ & $1,43 \mathrm{~d}$ & $2,75 \mathrm{a}$ & $1,99 \mathrm{c}$ & $2,43 \mathrm{ab}$ & $1,07 \mathrm{~d}$ & 10,1 \\
$0,20-0,40$ & $2,34 \mathrm{c}$ & $1,38 \mathrm{~d}$ & $3,18 \mathrm{a}$ & $2,82 \mathrm{~b}$ & $2,69 \mathrm{~b}$ & $1,37 \mathrm{~d}$ & 7,6 \\
$0-0,40$ & $6,86 \mathrm{~b}$ & $4,60 \mathrm{c}$ & $9,09 \mathrm{a}$ & $7,08 \mathrm{~b}$ & $8,47 \mathrm{a}$ & $5,22 \mathrm{c}$ & 9,0 \\
\hline & & \multicolumn{7}{c}{ Estoque de C-AF } \\
$0-0,05$ & $1,17 \mathrm{~cd}$ & $0,98 \mathrm{~d}$ & $1,76 \mathrm{a}$ & $1,33 \mathrm{bcd}$ & $1,35 \mathrm{bc}$ & $1,61 \mathrm{ab}$ & 13,6 \\
$0,05-0,10$ & $1,30 \mathrm{bc}$ & $1,05 \mathrm{c}$ & $2,00 \mathrm{a}$ & $1,49 \mathrm{~b}$ & $1,28 \mathrm{bc}$ & $1,38 \mathrm{bc}$ & 15,8 \\
$0,10-0,20$ & $2,80 \mathrm{~b}$ & $1,98 \mathrm{c}$ & $4,11 \mathrm{a}$ & $3,19 \mathrm{~b}$ & $2,81 \mathrm{~b}$ & $2,76 \mathrm{~b}$ & 12,6 \\
$0,20-0,40$ & $4,71 \mathrm{bc}$ & $3,15 \mathrm{~d}$ & $6,49 \mathrm{a}$ & $5,36 \mathrm{~b}$ & $4,53 \mathrm{bc}$ & $4,37 \mathrm{c}$ & 9,4 \\
$0-0,40$ & $9,98 \mathrm{~b}$ & $7,16 \mathrm{c}$ & $14,36 \mathrm{a}$ & $11,37 \mathrm{~b}$ & $9,97 \mathrm{~b}$ & $10,12 \mathrm{~b}$ & 8,6 \\
\hline
\end{tabular}

${ }^{(1)}$ Médias seguidas de letras iguais na linha, em cada camada, não diferem pelo teste de Tukey a $5 \%$ de probabilidade.
AH/AF diminuíram com o tempo de adoção do SPD. O mesmo padrão foi observado por Caetano et al. (2013) e Guareschi et al. (2013a), porém com valores dessa relação sempre superiores à unidade. A manutenção de maiores teores de $\mathrm{C}-\mathrm{AH}$, em relação aos teores de $\mathrm{C}-\mathrm{AF}$, evidencia solos preservados com melhoria dos atributos químicos (Canellas et al., 2003).

Os maiores estoques de C-AF ocorreram na área de $\mathrm{SPD}_{22}$ e chegaram a $6,49 \mathrm{Mg} \mathrm{ha}^{-1}$ na camada de 0,20 0,40 m, superiores aos observados nas demais áreas, em todas as camadas, com exceção da área de mata na camada de $0-0,05 \mathrm{~m}$ (Tabela 3 ). Esse resultado é confirmado pelo maior estoque dessa fração na seção de 0-0,40 m. Em função do maior estoque em $\mathrm{SPD}_{22}$, é evidente que sua $\triangle \mathrm{EC}$ em relação à área de referência será positiva e maior que a das demais áreas, como se pode observar na Figura $2 \mathrm{C}$.

Houve tendência de aumento nos teores (Tabela 2) e estoques (Tabela 3) de C-HUM com o tempo de implantação do SPD. Maiores teores e estoques de C-HUM indicam maior retenção de água, melhoria na agregação do solo (Benites et al., 2003) e maior retenção de cátions (Souza \& Melo, 2003). Na Figura 2 A, é possível observar variação negativa dos estoques de C-HUM em todas as áreas, com exceção da área de pastagem.

Com relação aos teores e estoques C-AH (Tabelas 2 e 3), destaca-se o aumento com o tempo de implantação do SPD em todas as camadas, principalmente quando se compara a área de $\mathrm{SPD}_{22} \mathrm{com}$ as demais. Os AH são considerados marcadores do processo de humificação, como tal, e refletem tanto a condição de gênese como de manejo do solo (Canellas et al., 2003). Os maiores teores e estoques de $\mathrm{C}-\mathrm{AF}$ e $\mathrm{C}-\mathrm{AH}$, na área de $\mathrm{SPD}_{22}$, indicam presença de $\mathrm{C}$ em frações de menor estabilidade. Para o estoque de $\mathrm{C}-\mathrm{AH}$, na seção de $0-0,40 \mathrm{~m}$, a área de $\mathrm{SPD}_{22}$ foi semelhante à área de pastagem cultivada por 38 anos (Tabela 3), com variação positiva em comparação à área de mata (Figura $2 \mathrm{~B}$ ).

Os teores de $\mathrm{C}$ das frações oxidáveis, bem como suas respectivas relações, podem ser utilizados para avaliar a dinâmica da matéria orgânica. Para a camada de 0-0,05 m, a área de mata apresentou maiores teores de $\mathrm{C}$ nas frações $\mathrm{F} 1$ (carbono lábil, $\mathrm{C}_{\mathrm{L}}$ ) e F3, com valores de 12,87 e $8,52 \mathrm{~g} \mathrm{~kg}^{-1}$, respectivamente (Tabela 3 ). Para a fração F2, as áreas foram semelhantes entre si, e chegaram a teores de $4,57 \mathrm{~g} \mathrm{~kg}^{-1}$. A área de pastagem,

Pesq. agropec. bras., Brasília, v.51, n.9, p.1529-1538, set. 2016 DOI: 10.1590/S0100-204X2016000900052 
nessa camada, apresentou maior teor de $\mathrm{C}$ na F4, ou seja, na fração mais recalcitrante.

As áreas de mata e pastagem apresentaram valores superiores especialmente das frações mais lábeis do C.
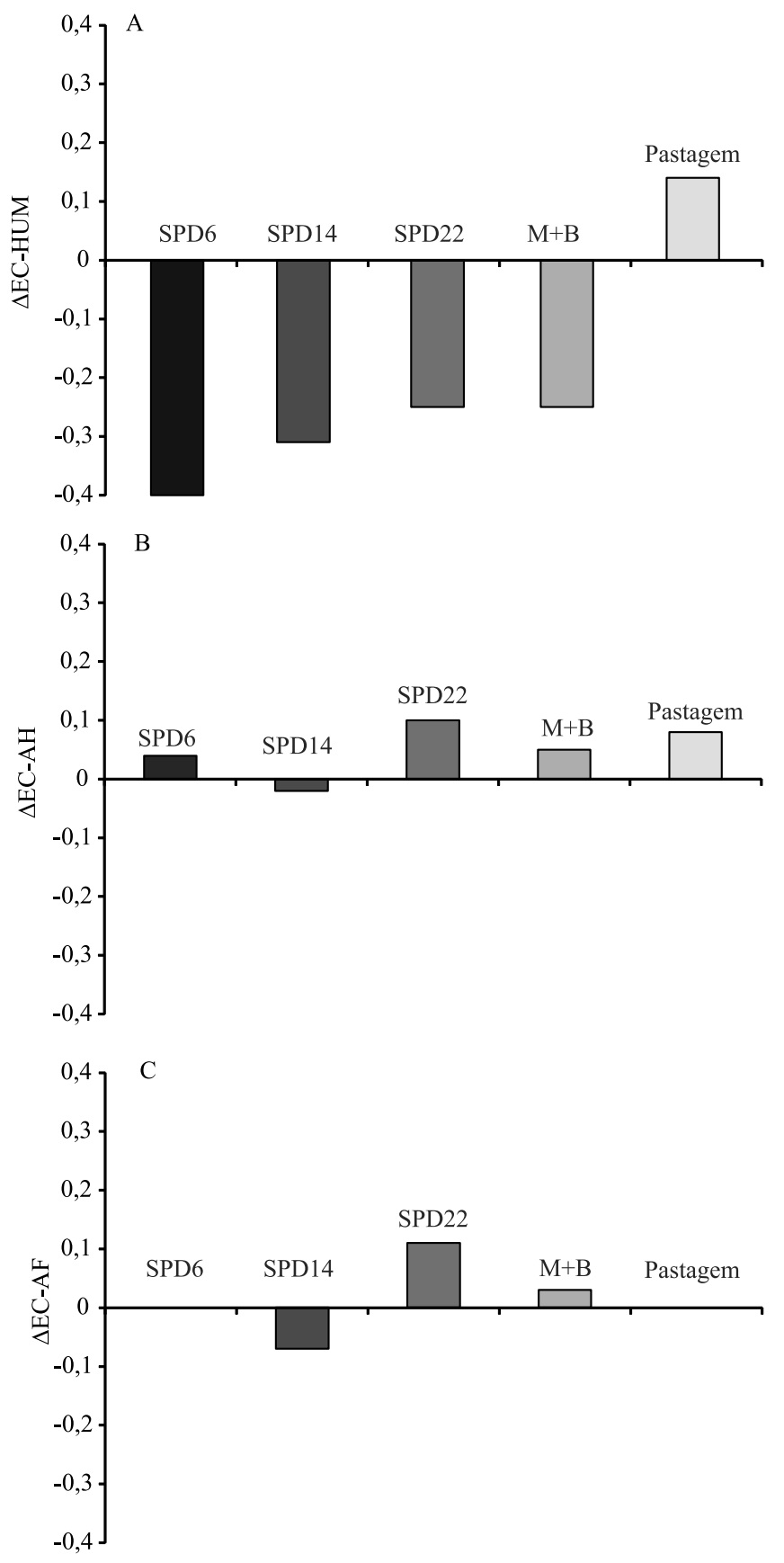

Figura 2. Variação do estoque de $\mathrm{C}(\Delta \mathrm{EC})$ das frações humificadas da matéria orgânica do solo das áreas manejadas na seção de $0-0,4 \mathrm{~m}$, em relação à área de mata nativa: $\mathrm{A}$, $\Delta \mathrm{EC}-\mathrm{HUM}$; B, $\Delta \mathrm{EC}-\mathrm{AH}$; e C, $\Delta \mathrm{EC}-\mathrm{AF}$.
Esse resultado está de acordo com os maiores teores de COT observados nessas áreas (Tabela 2). Guareschi et al. (2013b), ao avaliar uma cronossequência de SPD, relataram aumento nos teores da fração F1 com o tempo de implantação em todas as profundidades. Loss et al. (2013) observaram maior contribuição da fração F1 em área de SPD no Cerrado com o uso de maior número de culturas no sistema de manejo (girassol/milheto/soja e milho). A fração F1 pode ser considerada boa indicadora de sensibilidade da fração orgânica ao manejo do solo (Barreto et al., 2011), com diferenças visíveis mesmo em períodos mais curtos (Chan et al., 2001).

Para o $\mathrm{C}_{\mathrm{NL}}$, foram observadas maiores diferenças na camada de $0-0,05 \mathrm{~m}$, com maiores teores nas áreas de pastagem $\left(23,05 \mathrm{~g} \mathrm{~kg}^{-1}\right)$ e mata $\left(27,91 \mathrm{~g} \mathrm{~kg}^{-1}\right)$ (Tabela 4), e aumento nos teores com o tempo de implantação do SPD (6 e 22 anos), padrão concordante com os maiores teores de CT e C-HUM apresentados nessa camada (Tabela 2).

Com exceção da camada $0-0,05 \mathrm{~m}$, não foi observado aumento nos teores da fração F4 com o tempo de adoção do SPD, resultado discordante do relatado por Guareschi et al. (2013b). Na camada de 0-0,05 m, a área de pastagem apresentou maior teor dessa fração $\left(7,65 \mathrm{~g} \mathrm{~kg}^{-1}\right)$. Para a camada de $0,05-0,10$ $\mathrm{m}$, os maiores teores foram observados nas áreas de mata, pastagem e $\mathrm{SPD}_{14}$. Já na camada de $0,10-0,20$ $\mathrm{m}$, a fração $\mathrm{F} 3$, na área de pastagem $\left(6,73 \mathrm{~g} \mathrm{~kg}^{-1}\right)$, e $\mathrm{F} 4$, na $\mathrm{M}+\mathrm{B}\left(4,35 \mathrm{~g} \mathrm{~kg}^{-1}\right)$ apresentaram os maiores teores (Tabela 4). De modo geral, a área de pastagem destacou-se com maiores teores de $\mathrm{C}$ nas frações $\mathrm{F} 3$ e F4. Maiores teores de $\mathrm{C}$ nessas frações estão de acordo com os maiores teores de C-HUM (Tabela 2), fração química mais estável da MOS.

A distribuição relativa entre as frações lábeis e recalcitrantes da MOS é representada pelas relações $\mathrm{F} 1 / \mathrm{F} 4$ e F1+F2/F3+F4. De maneira geral, nas áreas de $\mathrm{SPD}$, a fração $\mathrm{F} 1$ predomina, em comparação à $\mathrm{F} 4$ (Tabela 4). Após 15 anos de sucessão soja/azevém sob SPD, Loss et al. (2014) relataram valores dessa relação entre 0,86 e 1,25 .

Quando as quatro frações foram avaliadas conjuntamente $(\mathrm{F} 1+\mathrm{F} 2 / \mathrm{F} 3+\mathrm{F} 4)$, a área de pastagem destacou-se, com valores próximos à unidade, especialmente nas duas primeiras camadas. Já para a relação $\mathrm{F} 1 / \mathrm{F} 4$, a área de $\mathrm{SPD}_{22}$ apresentou os maiores valores, entre 1,00 e 1,18, semelhantes aos observados 
na área de mata (Tabela 4). Esse resultado indica maior entrada de material vegetal de maior labilidade, o que também foi observado por Loss et al. (2013) em áreas de SPD e ILP no Cerrado goiano. Outro indicativo da entrada de $\mathrm{C}$ com maior labilidade nas áreas de $\mathrm{SPD}_{22}$ e $\mathrm{M}+\mathrm{B}$ é obtido pela avaliação da relação $\mathrm{C}_{\mathrm{L}} / \mathrm{C}$, em que essas áreas se destacaram na camada de $0-0,05 \mathrm{~m}$. Os valores dessa relação foram superiores aos obtidos por Rangel et al. (2008) e Guareschi et al. (2013b).

De maneira geral, a participação das frações F1 e F2 diminui em profundidade, tendo passado de 52 para 48 e $44 \%$, nas camadas de $0-0,05,0,05-0,10$ e $0,10-0,20$ $\mathrm{m}$, respectivamente. Já as frações F3 e F4 aumentaram de 48, para 52 e 56\% (Tabela 4). Guareschi et al. (2013b) observaram que a participação das frações mais recalcitrantes chegou a 66\%. Loss et al. (2013) encontraram valores da relação $\mathrm{F} 1 / \mathrm{F} 4$ de 8,4 e, para $\mathrm{F} 1+\mathrm{F} 2 / \mathrm{F} 3+\mathrm{F} 4$, superiores a 2,0 em áreas com SPD e ILP no Cerrado goiano.

$\mathrm{Na}$ análise canônica para as frações químicas da MOS, a primeira e a segunda variável canônica corresponderam a 93,86 e $4,06 \%$ da variação total, respectivamente (Figura $3 \mathrm{~A}$ ). Na mesma análise, as frações oxidáveis corresponderam a 82,95 e $12,70 \%$ da variação total (Figura $3 \mathrm{~B}$ ).

Para as frações químicas da MOS (Figura $3 \mathrm{~A}$ ), houve formação de três grupos distintos, um formado pelas áreas de $\mathrm{SPD}_{6}, \mathrm{SPD}_{14} \mathrm{e} \mathrm{M}+\mathrm{B}$, outro grupo pela área de $\mathrm{SPD}_{22}$, e um terceiro pelas áreas de pastagem e mata. As variáveis que mais contribuíram para a

Tabela 4. Frações oxidáveis de carbono orgânico (F1, F2, F3 e F4), carbono não-lábil ( $\left.\mathrm{C}_{\mathrm{NL}}\right)$, proporção de carbono lábil/C total $\left(\mathrm{C}_{\mathrm{L}} / \mathrm{C}\right)$ e relações entre as frações oxidáveis $(\mathrm{F} 1 / \mathrm{F} 4$ e F1+F2/F3+F4) nos diferentes sistemas de manejo, na região Oeste do Paraná(1).

\begin{tabular}{|c|c|c|c|c|c|c|c|c|}
\hline \multirow[t]{2}{*}{$\begin{array}{l}\text { Sistema de } \\
\text { manejo }\end{array}$} & 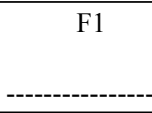 & --.-- & $\begin{array}{c}\mathrm{F} 3 \\
\left(\mathrm{~g} \mathrm{~kg}^{-1}\right) \\
\end{array}$ & ------- & -1------------ & $\begin{array}{c}\mathrm{C}_{\mathrm{L}} / \mathrm{C} \\
(\%) \\
\end{array}$ & $\mathrm{F} 1 / \mathrm{F} 4$ & $\begin{array}{l}\mathrm{F} 1+\mathrm{F} 2 \\
\mathrm{~F} 3+\mathrm{F} 4\end{array}$ \\
\hline & \multicolumn{5}{|c|}{$0-0,05 \mathrm{~m}$} & & & \\
\hline $\mathrm{SPD}_{6}$ & $5,52 \mathrm{e}$ & $4,06 \mathrm{a}$ & $4,78 \mathrm{~d}$ & $3,30 \mathrm{~d}$ & $13,26 \mathrm{~d}$ & $29,44 b c$ & $1,70 \mathrm{~b}$ & $1,19 \mathrm{a}$ \\
\hline $\mathrm{SPD}_{14}$ & $6,67 d$ & $2,40 \mathrm{c}$ & $5,54 \mathrm{c}$ & $5,74 \mathrm{~b}$ & $14,27 \mathrm{~cd}$ & $31,87 \mathrm{~b}$ & $1,16 \mathrm{c}$ & $0,80 \mathrm{c}$ \\
\hline $\mathrm{SPD}_{22}$ & $7,79 \mathrm{c}$ & $4,34 \mathrm{a}$ & $6,20 \mathrm{~b}$ & $4,05 \mathrm{c}$ & $16,23 \mathrm{c}$ & $32,55 \mathrm{ab}$ & $1,94 \mathrm{ab}$ & $1,18 \mathrm{a}$ \\
\hline $\mathrm{M}+\mathrm{B}$ & $6,93 \mathrm{~d}$ & $3,22 b$ & $5,83 \mathrm{bc}$ & $3,39 \mathrm{~cd}$ & $12,03 \mathrm{~d}$ & $36,68 \mathrm{a}$ & $2,05 \mathrm{ab}$ & $1,10 \mathrm{ab}$ \\
\hline Pastagem & $8,81 \mathrm{~b}$ & $4,24 \mathrm{a}$ & $5,82 \mathrm{bc}$ & $7,65 a$ & $23,05 b$ & $26,97 \mathrm{c}$ & $1,11 \mathrm{c}$ & $0,95 b c$ \\
\hline Mata & $12,87 \mathrm{a}$ & $4,57 \mathrm{a}$ & $8,52 \mathrm{a}$ & $5,74 \mathrm{~b}$ & $27,91 \mathrm{a}$ & $31,66 \mathrm{~b}$ & $2,26 a$ & $1,22 \mathrm{a}$ \\
\hline \multirow[t]{2}{*}{ CV(\%) } & 4,3 & 8,3 & 5,0 & 7,3 & 7,6 & 7,4 & 11,2 & 7,6 \\
\hline & \multicolumn{8}{|c|}{$0,05-0,10 \mathrm{~m}$} \\
\hline $\mathrm{SPD}_{6}$ & $3,96 \mathrm{e}$ & $3,10 \mathrm{~b}$ & $4,97 \mathrm{c}$ & $3,51 \mathrm{~b}$ & $11,94 b c$ & $24,93 b$ & $1,13 \mathrm{c}$ & $0,83 \mathrm{~b}$ \\
\hline $\mathrm{SPD}_{14}$ & $5,10 \mathrm{~cd}$ & $2,11 \mathrm{c}$ & $4,95 \mathrm{c}$ & $4,79 a$ & $11,66 \mathrm{c}$ & $30,41 \mathrm{a}$ & $1,08 \mathrm{c}$ & $0,74 \mathrm{~b}$ \\
\hline $\mathrm{SPD}_{22}$ & $5,46 b c$ & $3,26 \mathrm{~b}$ & $5,00 \mathrm{bc}$ & $2,78 \mathrm{c}$ & $12,79 b c$ & $30,03 a$ & $1,97 \mathrm{a}$ & $1,13 \mathrm{a}$ \\
\hline $\mathrm{M}+\mathrm{B}$ & $4,79 d$ & $2,22 \mathrm{c}$ & $5,69 \mathrm{~b}$ & $3,63 b$ & $12,53 b c$ & $27,73 \mathrm{ab}$ & $1,33 \mathrm{c}$ & $0,75 b$ \\
\hline Pastagem & $5,89 \mathrm{~b}$ & $3,12 b$ & $4,13 \mathrm{~d}$ & $4,49 a$ & $13,71 \mathrm{~b}$ & $30,12 \mathrm{a}$ & $1,32 \mathrm{c}$ & $1,05 \mathrm{a}$ \\
\hline Mata & $8,15 \mathrm{a}$ & $4,12 \mathrm{a}$ & $6,42 \mathrm{a}$ & $4,92 \mathrm{a}$ & $17,89 \mathrm{a}$ & $31,36 \mathrm{a}$ & $1,66 \mathrm{~b}$ & $1,08 \mathrm{a}$ \\
\hline \multirow[t]{2}{*}{ CV(\%) } & 5,5 & 8,1 & 6,9 & 7,8 & 7,2 & 7,2 & 10,1 & 7,2 \\
\hline & \multicolumn{8}{|c|}{$0,10-0,20 \mathrm{~m}$} \\
\hline $\mathrm{SPD}_{6}$ & $2,71 d$ & $1,92 \mathrm{c}$ & $3,92 \mathrm{~cd}$ & $3,54 \mathrm{~b}$ & $9,73 b c$ & $21,78 \mathrm{c}$ & $0,77 \mathrm{~d}$ & $0,62 \mathrm{~cd}$ \\
\hline $\mathrm{SPD}_{14}$ & $3,88 \mathrm{c}$ & $0,98 \mathrm{e}$ & $5,59 \mathrm{~b}$ & $2,97 \mathrm{bc}$ & $8,84 \mathrm{c}$ & $30,59 \mathrm{ab}$ & $1,31 \mathrm{c}$ & $0,57 \mathrm{~d}$ \\
\hline $\mathrm{SPD}_{22}$ & $5,08 \mathrm{a}$ & $1,54 \mathrm{~d}$ & $4,31 \mathrm{c}$ & $2,32 \mathrm{~cd}$ & $9,68 \mathrm{bc}$ & $34,89 \mathrm{a}$ & $2,23 a$ & $1,00 \mathrm{a}$ \\
\hline $\mathrm{M}+\mathrm{B}$ & $3,39 \mathrm{c}$ & $2,25 \mathrm{~b}$ & $3,71 \mathrm{~d}$ & $4,35 \mathrm{a}$ & $12,43 \mathrm{ab}$ & $21,93 \mathrm{c}$ & $0,78 \mathrm{~d}$ & $0,70 \mathrm{c}$ \\
\hline Pastagem & $5,50 \mathrm{a}$ & $3,00 \mathrm{a}$ & $6,73 a$ & $3,18 \mathrm{~b}$ & $14,32 \mathrm{a}$ & $27,75 b c$ & $1,77 \mathrm{~b}$ & $0,86 \mathrm{~b}$ \\
\hline Mata & $4,43 b$ & $1,99 \mathrm{~b}$ & $4,31 \mathrm{c}$ & $2,23 d$ & $10,51 b c$ & $29,84 \mathrm{ab}$ & $2,00 \mathrm{ab}$ & $0,98 \mathrm{a}$ \\
\hline$\overline{C V(\%)}$ & 5,9 & 8,2 & 6,0 & 11,3 & 14,1 & 11,8 & 15,6 & 5,5 \\
\hline
\end{tabular}


discriminação dos sistemas de manejo foram o carbono não humificado $(\mathrm{CNH})$ e a representatividade (\%) dos teores de AF e HUM em relação ao carbono total. $\mathrm{Na}$
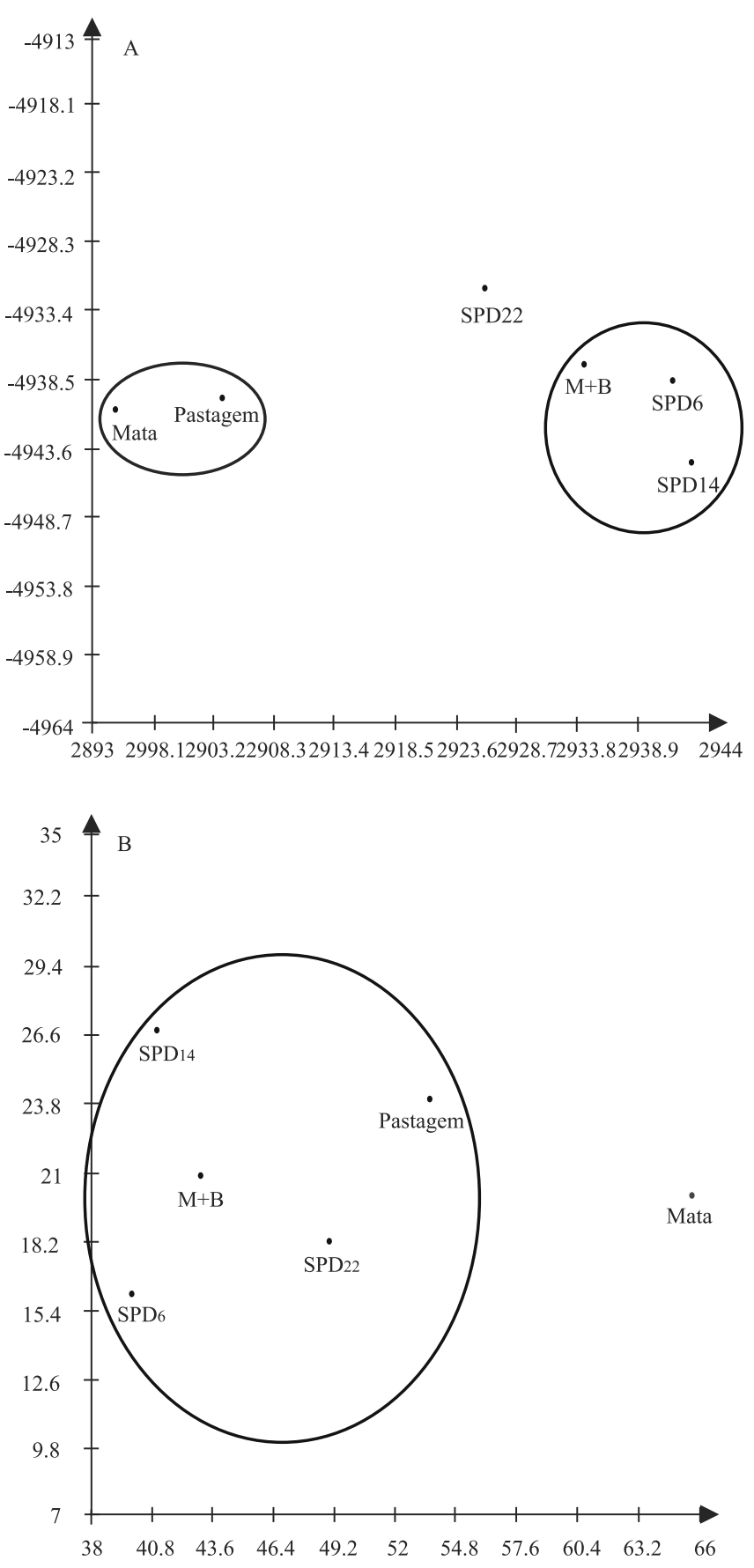

Figura 3. Dispersão dos diferentes sistemas de uso e manejo e agrupamento pelo método de Tocher modificado das duas primeiras variáveis canônicas em função das frações químicas (A) e oxidáveis (B) da matéria orgânica do solo. formação dos grupos em função das frações oxidáveis da MOS (Figura $3 \mathrm{~B}$ ), foram formados apenas dois grupos, um representado pela área de mata nativa, e outro, pelos sistemas manejados em SPD e pastagem, em que os teores de $\mathrm{CT}$ e $\mathrm{C}$ das frações $\mathrm{F} 1$ e F3 foram os atributos que mais contribuíram para discriminar os sistemas de manejo.

As frações químicas da MOS foram as que discriminaram de forma mais acentuada as áreas avaliadas em função do tempo de implantação e sistema de manejo. Trabalhos como os de Rossi et al. (2011) e Guareschi et al. (2013a) relatam a sensibilidade das frações químicas em detectar mudanças nos sistemas de manejo ao longo do tempo de condução.

\section{Conclusões}

1. O teor de carbono total, as frações mais lábeis da matéria orgânica e o estoque de carbono das frações humificadas aumentam em função do tempo de implantação do sistema plantio direto.

2. O não revolvimento do solo implica maior estabilidade do carbono, com predomínio da fração humina.

3. Há predomínio das frações lábeis da matéria orgânica em superfície, e das recalcitrantes nas camadas mais profundas.

4. As frações químicas da matéria orgânica do solo discriminam de forma mais sensível os sistemas de manejo avaliados.

\section{Agradecimentos}

À Coordenação de Aperfeiçoamento de Pessoal de Nível Superior (Capes), pela concessão de bolsas; e ao Conselho Nacional de Desenvolvimento Científico e Tecnológico (CNPq), pelo apoio financeiro e pela concessão de bolsas de produtividade.

\section{Referências}

ANGHINONI, I. Fertilidade do solo e seu manejo no sistema plantio direto. In: NOVAIS, R.F.; ALVAREZ V., V.H.; BARROS, N.F.; FONTES, R.L.F.; CANTARUTTI, R.B.; NEVES, J.C.L. Fertilidade do solo. Viçosa: Sociedade Brasileira de Ciência do Solo, 2007. p.873-928.

ASSIS, C.P. de; JUCKSCH, I.; MENDONÇA, E. de S.; NEVES, J.C.L. Carbono e nitrogênio em agregados de Latossolo submetido a diferentes sistemas de uso e manejo. Pesquisa Agropecuária 
Brasileira, v.41, p.1541-1550, 2006. DOI: 10.1590/S0100204X2006001000012.

BARRETO, A.C.; FREIRE, M.B.G.S.; NACIF, P.G.S.; ARAÚJO, Q.R.; FREIRE, F.J.; INÁCIO, E.S.B. Fracionamento químico e físico do carbono orgânico total em um solo de mata submetido a diferentes usos. Revista Brasileira de Ciência do Solo, v.32, p.1471-1478, 2008. DOI: 10.1590/S010006832008000400011.

BARRETO, P.A.B.; GAMA-RODRIGUES, E.F.; GAMARODRIGUES, A.C.; FONTES, A.G.; POLIDORO, J.C.; MOCO, M.K.S.; MACHADO, R.C.R.; BALIGAR, V.C. Distribution of oxidizable organic $\mathrm{C}$ fractions in soils under cacao agroforestry systems in Southern Bahia, Brazil. Agroforestry Systems, v.81, p.213-220, 2011. DOI: 10.1007/s10457-010-9300-4.

BENITES, V.M.; MÁDARI, B.; MACHADO, P.L.O.A. Extração e fracionamento quantitativo de substâncias húmicas do solo: um procedimento simplificado e de baixo custo. Rio de Janeiro: Embrapa Solos, 2003. 7p. (Embrapa Solos. Comunicado técnico, 16).

BEZERRA, R.P.M.; LOSS, A.; PEREIRA, M.G.; PERIN,A. Formas de carbono em latossolo sob sistemas de plantio direto e integração lavoura-pecuária no cerrado, Goiás. Semina: Ciências Agrárias, v.34, p.2637-2654, 2013. DOI: 10.5433/1679-0359.2013v34n 6p2637.

CAETANO, J.O.; BENITES, V. de M.; SILVA, G.P.; SILVA, I.R. da; ASSIS, R.L. de; CARGNELUTTI FILHO A. Dinâmica da matéria orgânica de um Neossolo Quartzarênico de cerrado convertido para cultivo em sucessão de soja e milheto. Revista Brasileira de Ciência do Solo, v.37, p.1245-1255, 2013. DOI: 10.1590/S0100-06832013000500014.

CAMPOS, L.P.; LEITE, L.F.C.; MACIEL, G.A.; BRASIL, E.L.; IWATA, B. de F. Estoques e frações de carbono orgânico em Latossolo Amarelo submetido a diferentes sistemas de manejo. Pesquisa Agropecuária Brasileira, v.48, p.304-312, 2013. DOI: 10.1590/S0100-204X2013000300009.

CANELLAS, L.P.; VELLOSO, A.C.X.; MARCIANO, C.R.; RAMALHO, J.F.G.P.; RUMJANEK, V.M.; REZENDE, C.E.; SANTOS, G.A. Propriedades químicas de um Cambissolo cultivado com cana-de-açúcar, com preservação do palhiço e adição de vinhaça por longo tempo. Revista Brasileira de Ciência do Solo, v.27, p.935-944, 2003. DOI: 10.1590/S010006832003000500018.

CARVALHO, J.L.N.; CERRI, C.E.P.; FEIGL, B.J.; PÍCCOLO, M.C.; GODINHO, V.P. CERRI, C.C. Carbon sequestration in agricultural soils in the Cerrado region of the Brazilian Amazon. Soil and Tillage Research, v.103, p.342-349, 2009. DOI: 10.1016/j.still.2008.10.022.

CAVIGLIONE, J.H.; KIIHL, L.R.B.; CARAMORI, P.H.; OLIVEIRA, D. de. Cartas climáticas do Paraná. Londrina: Iapar, 2000. 1 CD-ROM.

CHAN, K.Y.; BOWMAN, A.; OATES, A. Oxidizible organic carbon fractions and soil quality changes in an Paleustalf under different pasture leys. Soil Science, v.166, p.61-67, 2001. DOI: 10.1097/00010694-200101000-00009.
CRUZ, C. D. Programa genes: biometria. Viçosa: Ed. da UFV, 2006. 382p.

CRUZ, C. D.; REGAZZI, A. J. Modelos biométricos aplicados ao melhoramento genético. Viçosa: Ed. da UFV, 1994. 394p.

ELLERT, B.H.; BETTANY, J.R. Calculation of organic matter and nutrients stored in soils under contrasting management regimes. Canadian Journal of Soil Science, v.75, p.529-538, 1995. DOI: 10.4141/cjss95-075.

FOLEY, J.A.; DEFRIES, R.; ASNER, G.P.; BARFORD, C.; BONAN, G.; CARPENTER, S.R.; CHAPIN, F.S.; COE, M.T.; DAILY, G.C.; GIBBS, H.K.; HELKOWSKI, J.H.; HOLLOWAY, T.; HOWARD, E.A.; KUCHARIK, C.J.; MONFREDA, C.; PATZ, J.A.; PRENTICE, I.C.; RAMANKUTTY, N.; SNYDER, P.K. Global consequences of land use. Science, v.309, p.570-574, 2005. DOI: $10.1126 /$ science.1111772.

FONTANA, A.; PEREIRA, M.G.; LOSS, A.; CUNHA, T.J.F.; SALTON, J.C. Atributos de fertilidade e frações húmicas de um Latossolo Vermelho no Cerrado. Pesquisa Agropecuária Brasileira, v.41, p.847-853, 2006. DOI: 10.1590/S0100$204 X 2006000500018$.

GUARESCHI, R.F.; PEREIRA, M.G.; PERIN, A. Frações da matéria orgânica em áreas de Latossolo sob diferentes sistemas de manejo no Cerrado do estado de Goiás. Semina: Ciências Agrárias, v.34, p.2615-2628, 2013a. DOI: 10.5433/1679-0359.2013v34n $6 \mathrm{p} 2615$.

GUARESCHI, R.F.; PEREIRA, M.G.; PERIN, A. Oxidizable carbon fractions in Red Latosol under different management systems. Revista Ciência Agronômica, v.44, p.242-250, 2013 b. DOI: $10.1590 / \mathrm{S} 1806-66902013000200005$.

GUERRA, J.G.M.; SANTOS, G. de A.; SILVA, L.S. da; CAMARGO, F.A.O. Macromoléculas e substâncias húmicas. In: SANTOS, G. de A.; SILVA, L.S. da; CANELLAS, L.P.; CAMARGO, F.A.O. Fundamentos da matéria orgânica do solo: ecossistemas tropicais e subtropicais. 2.ed. Porto Alegre: Metrópole, 2008. p.19-25.

LOSS, A.; PEREIRA, M.G.; ANJOS, L.H.C. dos; FERREIRA, E.P.; BEUTLER, S.J.; SILVA, E.M.R. da. Oxidizable organic carbon fractions and soil aggregation in areas under different organic production systems in Rio de Janeiro, Brazil. Tropical and Subtropical Agroecosystems, v.14, p.699-708, 2011.

LOSS, A.; PEREIRA, M.G.; PERIN, A.; BEUTLER, S.J.; ANJOS, L.H.C. dos. Oxidizable carbon and humic substances in rotation systems with brachiaria/livestock and pearl millet/no livestock in the Brazilian Cerrado. Spanish Journal of Agricultural Research, v.11, p.217-231, 2013. DOI: 10.5424/sjar/2013111-3416.

LOSS, A; PEREIRA, M.G.; COSTA, E.M.; BEUTLER, S.J. Frações granulométricas e oxidáveis de matéria orgânica sob diferentes sistemas de uso do solo, no Paraná, Brasil. Bioscience Journal, v.30, p.43-54, 2014.

RANGEL, O.J.P.; SILVA, C.A.; GUIMARÃES, P.T.G.; GUILHERME, L.R.G. Frações oxidáveis do carbono orgânico de Latossolo cultivado com cafeeiro em diferentes espaçamentos de plantio. Ciência e Agrotecnologia, v.32, p.429-437, 2008. DOI: 10.1590/S1413-70542008000200013. 
ROSSET, J.S.; LANA, M. do C.; PEREIRA, M.G.; SCHIAVO, J.A.; RAMPIM, L.; SARTO, M.V.M.; SEIDEL, E.P. Carbon stock, chemical and physical properties of soils under management systems with different deployment times in western region of Paraná, Brazil. Semina: Ciências Agrárias, v.35, p.3053-3072, 2014. DOI: 10.5433/1679-0359.2014v35n6p3053.

ROSSI, C.Q.; PEREIRA, M.G.; GIACOMO, S.G.; BETTA, M.; POLIDORO, J.C. Frações húmicas da matéria orgânica do solo cultivado com soja sobre palhada de braquiária e sorgo. Bragantia, v.70, p.622-630, 2011.DOI: 10.1590/S0006-87052011000300018.

SANTOS, H.G. dos; JACOMINE, P.K.T.; ANJOS, L.H.C. dos; OLIVEIRA, V.A. de; LUMBRERAS, J.F.; COELHO, M.R.;

ALMEIDA, J.A. de; CUNHA, T.J.F.; OLIVEIRA, J.B. de. Sistema brasileiro de classificação de solos. 3.ed. rev. e ampl. Brasília: Embrapa, 2013. 353p.
SISTI, C.P.J.; SANTOS, H.P. dos; KOHHANN, R.; ALVES, B.J.R.; URQUIAGA, S.; BODDEY, R.M. Change in carbon and nitrogen stocks in soil under 13 years of conventional or zero tillage in southern Brazil. Soil and Tillage Research, v.76, p.3958, 2004. DOI: 10.1016/j.still.2003.08.007.

SOUZA, W.J.O.; MELO, W.J. Matéria orgânica em um Latossolo submetido a diferentes sistemas de produção de milho. Revista Brasileira de Ciência do Solo, v.27, p.1113-1122, 2003. DOI: 10.1590/S0100-06832003000600016.

SWIFT, R.S. Organic matter characterization. In: SPARKS, D.L.; PAGE, A.L.; HELMKE, P.A.; LOEPPERT, R.H.; SOLTANPOUR, P.N.; TABATABAI, M.A.; JOHNSTON, C.T.; SUMNER, M.E. (Ed.). Methods of soil analysis. Madison: American Society of Agronomy, Soil Science Society American, 1996. p.1011-1020. (SSA book series, 5).

Recebido em 15 de junho de 2015 e aprovado em 10 de fevereiro de 2016 